\title{
Severity of imported falciparum malaria: effect of taking antimalarial prophylaxis
}

\author{
Stephen J Lewis, Robert N Davidson, Emma J Ross, Anthony P Hall
}

\begin{abstract}
Objective-To investigate the effects of antimalarial chemoprophylaxis and other variables on the severity of falciparum malaria.

Design-Review of consecutive malaria cases between 1987 and 1991 .

Setting-The Hospital for Tropical Diseases, London.

Subjects -250 consecutive cases of mild and 51 consecutive cases of severe falciparum malaria.

Results-Prophylaxis was taken in 52.4\% (131/ $250)$ of the cases of mild malaria and $21.6 \%(11 / 51)$ of cases of severe malaria. Severe malaria was more common in white patients than in those of African origin and was also seen more commonly in people returning from central, southern, and east Africa than in those returning from west Africa. Patients with severe malaria presented sooner than patients with mild malaria.
\end{abstract}

Conclusions-Prior chemoprophylaxis led to a reduction in the severity of falciparum malaria. Ethnic origin, time to presentation, and sex were also associated with the severity of malaria.

\section{Introduction}

The failure rates of antimalarial chemoprophylaxis are well documented. ${ }^{12}$ Deaths from malaria among travellers are known to be related to delays in treatment and failure to take antimalarial prophylaxis. The mortality from malaria imported into the United Kingdom was about $0 \cdot 8 \%$ for the period 1986-8. There is some evidence that severe falciparum malaria tends to occur less frequently in people of African origin ${ }^{4}$ and more commonly in patients not taking chemoprophylaxis. ${ }^{5}$

The aim of our study was to define those factors influencing the severity of malaria, especially the taking of chemoprophylaxis. The Hospital for Tropical Diseases treats over 300 cases of malaria annually. Patients are mainly self referred or referred by a general practitioner.

\section{Methods}

We reviewed 250 consecutive cases of mild falciparum malaria (January 1990 to August 1991) presenting to the Hospital for Tropical Diseases, London, and 51 consecutive cases of severe falciparum malaria collected over the period 1987-91. The notes were analysed for the taking of chemoprophylaxis (yes or no; partial compliance was classified as ineffectual - that is, no), age, sex, ethnic origin (white, African living in

BMF 1992;305:741-3 the United Kingdom, African living in Africa, and other), area of infection (west, east, central, or southern Africa and other), type of malaria (mild or severe), and time from onset of symptoms to presentation. As all patients with a history of fever had blood examined for malaria parasites immediately time to presentation was effectively the same as time to diagnosis. Severe malaria was defined according to World Health Organisation (1990) criteria $^{6}{ }^{6}$ In our series these patients predominantly had hyperparasitaemia ( $>5 \%$ of erythrocytes parasitised), cerebral malaria, or renal failure. Mild malaria was defined by the absence of the WHO criteria for severe malaria.

The data were obtained from the inpatient notes and report forms submitted to the Public Health Laboratory Service Malaria Reference Laboratory, which are routinely completed after each patient's discharge. As our computerised records of admissions before 1990 were incomplete we extended the search backwards to 1987 , until $>50$ severe cases were found. During the period 1987-91 the drugs recommended for chemoprophylaxis had not changed, so we felt no systematic bias would be introduced by doing this.

Statistical methods-The continuous variables age and time to presentation were analysed by Student's $t$ test. For the comparison of two proportions 95\% confidence intervals were calculated for the difference in the observed proportions. The $\chi^{2}$ test was used to detect an association between categorical variables. Forward stepwise logistic regression analysis was performed to model the odds of having severe malaria compared with mild malaria given six potential explanatory variables: age, sex, chemoprophylaxis, ethnic origin, time to presentation, and where the malaria was acquired.

\section{Results}

Data were obtained on 301 patients. The demographic characteristics are shown in table I. A number of different antimalarial regimens were used, though $96 \%$ of patients taking antimalarials took chloroquine or proguanil or a combination of the two.

There was no significant difference between the mild and severe groups with respect to age $(t=1 \cdot 06$, $\mathrm{p}=0 \cdot 29)$. The severe group presented earlier than the mild group $(t=5 \cdot 77, \mathrm{p}<0 \cdot 001)$. The $95 \%$ confidence interval for the difference in the observed proportions showed an association between severe malaria and no chemoprophylaxis ( $95 \%$ confidence interval $17 \%$ to $44 \%)$.

When ignoring the severity of malaria there was a statistically significant association between chemoprophylaxis uptake and time to presentation, patients taking chemoprophylaxis presenting later $(p=0 \cdot 011)$ (table II). Within the severe and mild subgroups there was no difference in the time to presentation between subjects who did and did not take chemoprophylaxis. There was no difference in the time to presentation among the different ethnic categories.

No association was suggested between severity of malaria and sex $(95 \%$ confidence interval $-0.4 \%$ to $18 \%$ ), but after controlling for the severity of malaria
Mea (female/male) No (\%) taking chemoprophylaxis

Mean days to presentation $(95 \% \text { confidence interval })^{\star}$

^Data available on 240 in mild group and 43 in severe group. 5 $30 \cdot 3(28 \cdot 8$ to 31.9$) \quad 32.5(28 \cdot 3$ to $36 \cdot 7)$ $131(52 \cdot 4) \quad 32 \cdot 5(28 \cdot 3 \cdot 6)$ $3 \cdot 8(3 \cdot 1$ to $4 \cdot 4)$ 
there was a weak association between sex and chemoprophylaxis uptake within the severe group $(p=0.068)$ but no association within the mild group $(\mathrm{p}=0.798)$ (table III).

To investigate the associations among ethnic origin, region where malaria was acquired, and severity of malaria 21 patients in the "other" categories were excluded because of small numbers. Africans living in Africa and those living in the United Kingdom were combined to form one group as they appeared to have the same proportions of severe versus mild malaria.

Fewer than $10 \%$ of Africans living in Africa took chemoprophylaxis compared with $50 \%$ of Africans living in the United Kingdom and almost $70 \%$ of white patients. Controlling for whether or not chemoprophylaxis was taken, there was a strong association between severity of malaria and ethnic group. Among people not taking chemoprophylaxis white patients were more likely than Africans to have severe malaria $\left(\chi^{2}=25.64\right.$ on 1 degree of freedom, $\left.p<0.0001\right)$. This difference was not significant when comparing both groups who took chemoprophylaxis (Fisher's exact test: $p=0.526$ ) (table IV). Severe malaria was seen as a greater proportion of malaria acquired in central and southern Africa (26\%) and east (22\%) than west (12\%).

To investigate further the interrelations between variables, by using the $5 \%$ level of statistical significance to decide whether to retain a variable in the analysis, modelling was performed by forward stepwise multiple logistic regression with generalised linear interactive modelling. This identified chemoprophylaxis uptake (yes or no), ethnic origin (African or white), time to presentation ( $<7$ days or $\geqslant 7$ days), and sex (male or female) as the best predictors of whether a patient had severe or mild malaria $(p<0.0001)$

In this model the odds of severe malaria decreased when chemoprophylaxis was used, patients not taking chemoprophylaxis being 10.5 times as likely to develop severe malaria as those taking chemoprophylaxis. The odds of a white patient developing severe malaria were eight times those of an African, and the odds of a patient presenting within seven days having severe malaria were $4 \cdot 2$ times those of patients presenting at seven days or more. Women were twice as likely as men to develop severe malaria (table V).

The proportions predicted and observed in the model are presented in table VI. White women not taking chemoprophylaxis who presented within seven days were most likely to have severe malaria. The model did not fit well for white women not taking chemoprophylaxis who presented at seven days or more: it underestimated their chances of having severe

TABLE II-Chemoprophylaxis uptake and time to presentation. Figures are numbers of patients

\begin{tabular}{lcc}
\hline & \multicolumn{2}{c}{ Time to presentation } \\
\cline { 2 - 3 } & $<7$ Days & $\geqslant 7$ Days \\
\hline No prophylaxis & 111 & 33 \\
Took prophylaxis & 87 & 52 \\
\hline Total & 198 & 85 \\
\hline
\end{tabular}

$\chi^{2}=6 \cdot 4, p=0 \cdot 011$

Data based on 283 patients for whom duration of symptoms was recorded

TABLE III-Use of antimalarial chemoprophylaxis by sex, controlling for severity of malaria

\begin{tabular}{lccccc}
\hline & \multicolumn{2}{c}{ Mild malaria } & & \multicolumn{2}{c}{ Severe malaria } \\
\cline { 2 - 3 } \cline { 5 - 6 } & Chemoprophylaxis & $\begin{array}{c}\text { No } \\
\text { chemoprophylaxis }\end{array}$ & & Chemoprophylaxis & $\begin{array}{c}\text { No } \\
\text { chemoprophylaxis }\end{array}$ \\
\hline No of males & 90 & 79 & 9 & 18 \\
No of females & 41 & 119 & 2 & 11 \\
\hline Total & 131 & $\chi^{2}=0.065, \mathrm{p}=0.798$ & & $\chi^{2}=3.33, \mathrm{p}=0.068$ \\
\hline
\end{tabular}

TABLE IV-Severity of malaria by ethnic group, controlling for use of chemoprophylaxis. Figures are numbers in each ethnic group

\begin{tabular}{lcccccc}
\hline & \multicolumn{2}{c}{ No prophylaxis } & & \multicolumn{2}{c}{ Took prophylaxis } \\
\cline { 2 - 3 } \cline { 6 - 7 } & Mild & Severe & & Mild & Severe \\
\hline African groups combined & 94 & 15 & & 51 & 3 \\
White & 17 & 22 & & 71 & 7 \\
\hline Total & 111 & 37 & & 122 & 10
\end{tabular}

TABLE V - Logistic regression model for severe malaria

\begin{tabular}{lc}
\hline Variable & Odds ratio $(95 \%$ confidence interval $)$ \\
\hline Chemoprophylaxis (no $v$ yes) & $10.52(3.52$ to 31.43$)$ \\
Ethnic origin (white $v$ African) & $8.04(3.15$ to 20.50$)$ \\
Time to presentation $(<7$ days $v$ & $4 \cdot 16(1.36$ to 12.70$)$ \\
$\quad \geqslant 7$ days) & $2 \cdot 12(0.97$ to 4.61$)$ \\
$\begin{array}{l}\text { Sex (female } v \text { male) } \\
\text { Interaction (prophylaxis by ethnic }\end{array}$ & $5.28(0.94$ to $29 \cdot 49)$ \\
\hline
\end{tabular}

TABLE VI - Proportion predicted (proportion observed) of white and African groups with severe malaria. Figures are percentages

\begin{tabular}{lccccc}
\hline & \multicolumn{2}{c}{ No prophylaxis } & & \multicolumn{2}{c}{ Took prophylaxis } \\
\cline { 6 - 6 } \cline { 5 - 6 } & White & African & & White & African \\
\hline Female: & & & & \\
$\quad$ Symptoms $<7$ days & $68(67)$ & $21(29)$ & & $17(10)$ & $20(8)$ \\
$\quad$ Symptoms $\geqslant 7$ days & $34(50)$ & $6(0)$ & & $5(0)$ & $3(0)$ \\
$\begin{array}{l}\text { Male: } \\
\text { Symptoms }<7 \text { days }\end{array}$ & $51(50)$ & $11(9)$ & & $9(10)$ & $6(6)$ \\
$\quad$ Symptoms $\geqslant 7$ days & $20(20)$ & $3(0)$ & & $2(6)$ & $2(6)$ \\
\hline
\end{tabular}

malaria. The model also did not perform well for African women taking chemoprophylaxis who presented within seven days: it overestimated their chances of developing severe malaria. However, the model seemed reasonable to describe the data and showed an association between use of chemoprophylaxis, ethnic origin, and the development of severe malaria, with a much higher proportion of white people than Africans developing severe malaria when chemoprophylaxis was not taken but similar proportions of the two ethnic groups developing severe malaria when chemoprophylaxis was taken.

\section{Discussion}

This study suffered from a major limitation inherent in any retrospective analysis, in that data were not originally recorded with this type of study in mind. The numbers of cases were not great, and data on duration of symptoms before presentation were not recorded in every case. We considered it unreliable to estimate the degree of compliance with chemoprophylaxis, and, in addition, more than one antimalarial regimen was in use. None the less, it appears that breakthrough attacks of falciparum malaria are significantly milder in patients who have taken chemoprophylaxis. Such information is highly relevant in choosing the best drug or drug combination for chemoprophylaxis, as well as the more usual considerations of failure rate in a given region, toxicity, and cost. A study comparing self reported compliance with antimalarial prophylaxis and blood concentrations of antimalarial drugs has shown that patients significantly overestimate their degree of compliance. ? Our study relied on the patients' account of the use of chemoprophylaxis and thus may have underestimated the full effect antimalarials could have in a fully compliant population. However, because our study reflects the impact under field conditions the data are perhaps of more practical use than data collected under clinical trial conditions.

Interestingly, Africans not taking chemoprophylaxis had significantly less risk of severe malaria despite being resident in the United Kingdom and thus 
having lost some of their acquired immunity to malaria. This suggests that there may be some genetic advantage in this ethnic group. When chemoprophylaxis was taken the proportions with severe and mild malaria were almost equal in white people and Africans. Severe malaria was associated with a shorter duration of symptoms before presentation than mild malaria, suggesting that a severe attack of malaria may be essentially different from a mild attack and not merely a mild attack in which the symptoms have been neglected or ignored. This in turn may reflect different host-parasite interactions. It is of interest that surveys of fatalities have highlighted late diagnosis, as distinct from late presentation, as a major factor contributing to death. ${ }^{8}$

A greater proportion of patients taking chemoprophylaxis presented after a week or longer of symptoms, which could mean that their symptoms were initially milder. The highest proportion of severe to mild malaria was seen in southern and central and east Africa, but this was accounted for by the fact that this was the area predominantly visited by white people. Though severe malaria was relatively less commonly acquired in west Africa, this was due to the fact that most travellers to or from this region were Africans either visiting relatives in the United Kingdom or living in the United Kingdom and visiting relatives in west Africa. A greater proportion of women acquired severe malaria but women within the severe group had a lower uptake of chemoprophylaxis than men. Within the mild group there was no difference in the proportion of men and women taking chemoprophylaxis. A larger, prospective study would be needed to assess and confirm the results of our study.

Particularly worthy of further study are the risk of severe malaria in different endemic areas, the effects of full versus partial drug compliance, and a comparison of different prophylactic regimens.

The finding that in many cases apparent failure of chemoprophylaxis is, rather, a partial success should enhance the confidence which both doctors and travellers alike may place in antimalarial prophylaxis.

We thank the Public Health Laboratory Service Malaria Reference Laboratory at the London School of Hygiene and Tropical Medicine for assistance in providing patient data.

1 Lackritz EM, Lobel HO, Howell J, Boland P, Campbell CC. Imported Plasmodium falciparum malaria in American travellers to Africa. $\mathscr{f} A M A$ 1991;265:383-5.

2 Phillips-Howard PA, Radalowicz A, Mitchell J, Bradley DJ. Risk of malaria in British residents returning from malarious areas. $B M \mathcal{F}$ 1990;300:499-503.

3 Bradley DJ. Current trends in malaria in Britain. $\mathcal{F} R$ Soc Med 1989;82 (suppl 17): $8-13$.

4 Hall AP, Canfield CJ. Resistant falciparum malaria in Vietnam: its rarity in Negro soldiers. Proceedings of the Helminthological Society of Washington 1972;39:66-70

5 Westeyn JCFM, DeGeus A. Chloroquine resistant falciparum malaria imported into the Netherlands. Bull World Health Organ 1985;63:101-8.

6 Warrell DA, Molyneux ME, Beales PF, eds. Severe and complicated malaria. 2nd ed. World Health Organization division of control of tropical diseases. Trans $R$ Soc Trop Med Hyg 1990;84(suppl 2): 1-65.

7 Behrens RH, Pryce DI, Taylor RB, Low ST. Comparison of reported compliance to plasma levels of chemoprophylactic drugs in patients with malaria. In: Lobel HO, ed. Proceedings of 2 nd international conference on travel medicine, Atlanta, Georgia, 1991. In press.

8 Greenburg AE, Lobel HO. Mortality from Plasmodium falciparum malaria in travellers from the United States, 1959 to 1987. Ann Intern Med 1990;113: 326-7.

(Accepted 23 fuly 1992)

\section{Cost of urology: financial audit in a clinical department}

\section{Peter M Cuckow}

Abstract

Objectives - To cost a clinical unit over one month in 1991, to cost treatment of individual patients from audit data, and to compare this costing method with the hospital charging system.

Design-A financial breakdown was obtained for one month's work. Ward stay, operating time, investigations, and outpatient visits were costed and a formula (episode $=$ days on ward +hours of operating + investigations + outpatient visits) was used to cost patient episodes from audit data.

Setting-The adult urology unit in a teaching hospital.

Main outcome measures-Costs for each part of patients' treatment.

Results - Total cost was $£ 147796$ for 159 admissions, 738 inpatient days, 131 operations in 29 operating lists, and 615 outpatient visits. An uncomplicated transurethral prostatectomy cost $£ 1140$ but complications increased this to $£ 1500$ in another patient. The costs of diagnostic cystoscopy were $£ 130$ in outpatients, $£ 240$ in day surgery, and $£ 430$ in inpatients. Hospital charges do not reflect the individual costs of treatment, charges being greater than costs for some patients and lower than costs for others.

Conclusions-Clinicians can produce a financial analysis of their work and cost their patients' treatment. Audit is strongly advocated as a resource planning tool.

Department of Urology, Cambridge CB2 2QQ Peter $M$ Cuckow, research registrar

\section{Introduction}

Never have doctors needed greater financial insight. BMf 1992;305:743-6 service and change medical practice, although they are often inaccurate, not specific to an individual practice, and difficult to understand. I did a study to cost fully adult urology over one month at my hospital and answer the following questions: Can a clinician cost his or her unit? How much does the unit cost? How can this knowledge be applied? A costing formula was developed which allowed treatment of individual patients to be costed and provided further financial insights.

\section{Methods}

The study was conducted over 31 days (29 January to 28 February 1991) and a review of audit data showed that workload was comparable with that in previous months. The unit was divided into five areas to study use of resources: the ward, the operating theatre, the day surgery unit, outpatients, and investigations.

Most of the data required were already collected, manually or on computer, by the hospital so prospective data collection was unnecessary. Some laboratories with advanced costing systems could derive a bill for each consultant. For other areas Körner data, budgets, payroll information, and hospital price lists were used to derive costs.

Use of equipment and materials was determined from budget statements. Assessing each item separately, as done in my previous study, ${ }^{1}$ was considered too time consuming for the small costs incurred. More time was spent accounting for staff use. Staff costs were apportioned according to the amount of time spent working in the unit. Workload and patient information were available from computerised audit and the hospital's case mix system. 